\title{
Learning Visuomotor Policies with Deep Movement Primitives
}

\author{
Michail Theofanidis \\ Boston University \\ Boston, USA \\ theofan@bu.edu \\ Joe Cloud \\ University of Texas at Arlington \\ Arlington, USA \\ joe.cloud@uta.edu
}

\author{
Asil Kaan Bozcuoğlu \\ Institute for Artificial Intelligence \\ (IAI) \\ Bremen, Germany \\ asil@uni-bremen.de \\ Maria Kyrarini \\ University of Texas at Arlington \\ Arlington, USA \\ maria.kyrarini@uta.edu \\ Michael Beetz \\ Institute for Artificial Intelligence \\ (IAI) \\ Bremen, Germany \\ beetz@uni-bremen.de
}

Michael Neumann

University of Texas at Arlington

Bremen, Germany

joe.cloud@uta.edu

Fillia Makedon

University of Texas at Arlington

Arlington, USA

makedon@uta.edu

\begin{abstract}
In this paper, we present a novel method to learn end-to-end visuomotor policies for robotic manipulators. The method computes state-action mappings in a supervised learning manner from video demonstrations and robot trajectories. We show that the robot learns to perform different tasks by associating image features with the corresponding movement primitives of different grasp poses. To evaluate the effectiveness of the proposed learning method, we conduct experiments with a PR2 robot in a simulation environment. The purpose of these experiments is to evaluate the system's ability to perform manipulation tasks.
\end{abstract}

\section{CCS CONCEPTS}

- Computing methodologies $\rightarrow$ Supervised learning by classification.

ACM Reference Format:

Michail Theofanidis, Asil Kaan Bozcuoğlu, Michael Neumann, Joe Cloud, Maria Kyrarini, Fillia Makedon, and Michael Beetz. 2021. Learning Visuomotor Policies with Deep Movement Primitives. In The 14th PErvasive Technologies Related to Assistive Environments Conference (PETRA 2021), fune 29-fuly 2, 2021, Corfu, Greece. ACM, New York, NY, USA, 7 pages. https://doi.org/10.1145/3453892.3453899

In recent years, roboticists put substantial effort into building autonomous agents by leveraging the abilities of learning into the classical sense-reason-act loop [19]. Early research in the field of Human-Robot-Interaction (HRI) introduced the notion that robots

Permission to make digital or hard copies of all or part of this work for personal or classroom use is granted without fee provided that copies are not made or distributed for profit or commercial advantage and that copies bear this notice and the full citation on the first page. Copyrights for components of this work owned by others than ACM must be honored. Abstracting with credit is permitted. To copy otherwise, or republish, to post on servers or to redistribute to lists, requires prior specific permission and/or a fee. Request permissions from permissions@acm.org.

PETRA 2021, June 29-fuly 2, 2021, Corfu, Greece

(c) 2021 Association for Computing Machinery.

ACM ISBN 978-1-4503-8792-7/21/06 . .\$15.00

https://doi.org/10.1145/3453892.3453899

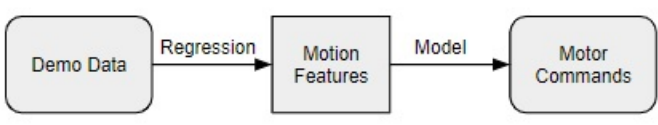

Figure 1: LfD with DMPs.

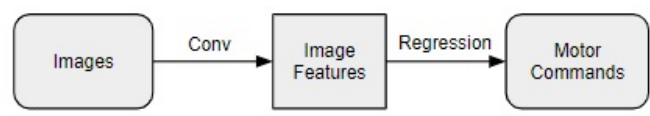

Figure 2: LFD with CNNs.

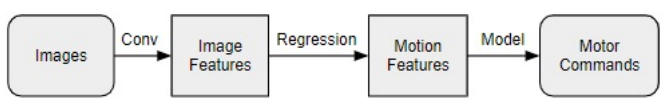

Figure 3: Proposed LfD method.

should learn to perform tasks through human guidance and not programming. This idea is influenced by the human tendency to learn practical skills by observing and imitating other humans [20], [3]. This trend established the area of imitation learning, which leads to the development of Learning from Demonstration (LfD) approaches [1].

LfD algorithms operate in two stages [1], [20], [2]; the demonstration and the execution stage. In the demonstration stage, the LfD method records data that associate with the world state and the action of the demonstrator. The learner then creates a stateaction mapping which accomplishes the demonstrated task. This mapping is often referred to in LfD literature as a policy. In the execution stage, the robot applies the policy to perform the task in a similar setting. LfD methods can be categorized according to how 


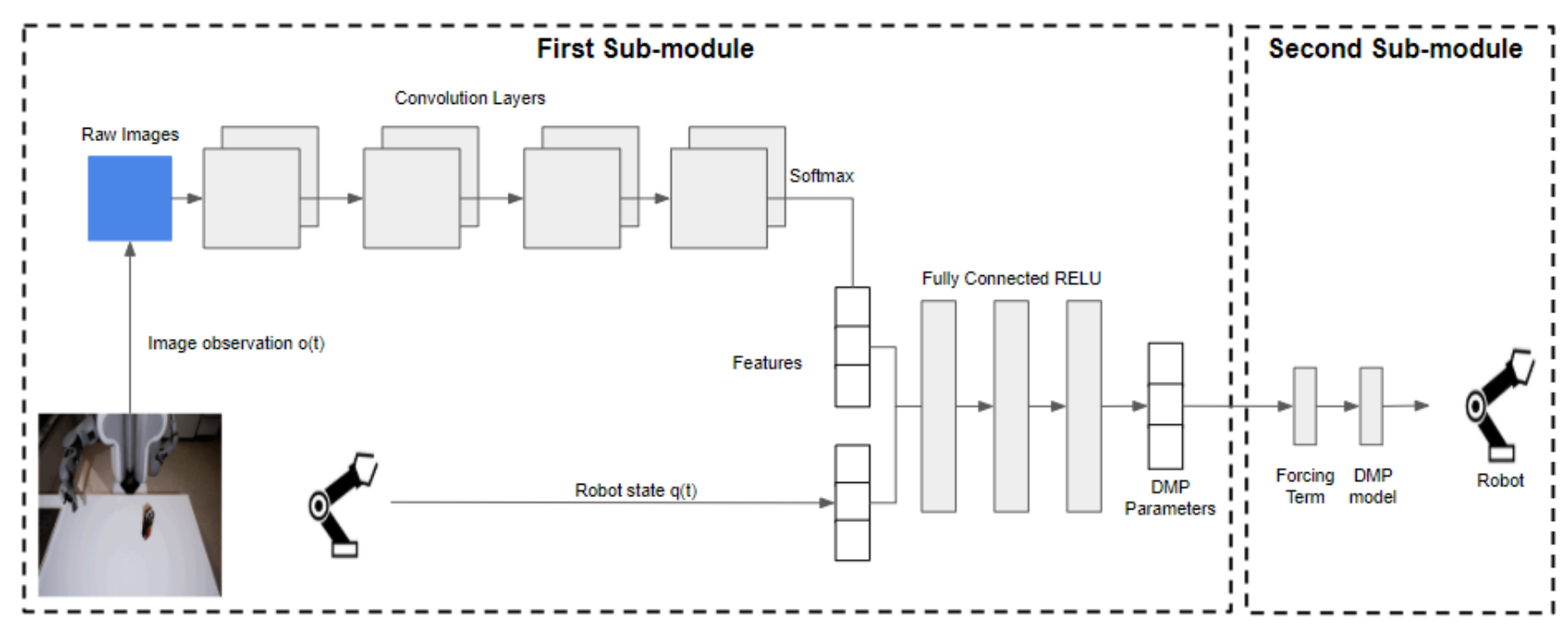

Figure 4: Proposed Learning Method Architecture.

the data is collected, what features are selected, and how the policy is derived [20], [1].

In recent years, many LfD frameworks have been applied to solve the problem of deriving policies that generalize the behavior of a robot over different state-action mappings. The development of the Dynamic Movement Primitive (DMP) framework [9] is an essential contribution to this trend, as it provides an abstraction layer that connects the dimensions of state, action, and environment by computing a motor policy with distinct meta-parameters that affect the behavior of the system [11]. However, the experiments reported in [14], [15], [16] have shown that tasks which are composed of a plethora of different sequences of kinematic configurations require multiple DMP policies to be learned. A typical test case for multiple DMPs is a robot learning tennis by demonstration [14], [15]. Muelling et al. proposed to solve the issue of motor primitives generalization by combining the meta-parameters of multiple DMP policies with a gating network.

With the development of modern Convolutional Neural Networks (CNNs), researchers began to develop systems that train on perception and control data to learn end-to-end policies from raw images to low-level motor commands [12], [13], such as joint torques. LfD systems that incorporate CNNs have the ability to learn policies from datasets with videos of demonstrations and their respective joint motor commands [5]. Since CNNs have the capacity to extrapolate visual information on a higher abstract dimension with respect to the presented data [4], LfD methods which combine CNNs can learn to perform tasks from different embodiment [6]. Such is the case when the authors of [21] learn tasks from both human and robot demonstrations to infer a policy of a new task from just one new human demonstration.

Recently, [7], [17] present methodologies that use both CNNs and DMPs. Specifically, the authors of [7] augment the learning capabilities of the DMP framework by creating a deep encoderdecoder network that maps raw images to the parameters of a DMP policy. Since the meta-parameters of a DMP policy lie in different dimensions, a custom cost function is used to train the network. Similarly, [17] proposes a two-step learning method that combines CNNs and DMPs. In the first step, a CNN learns to map images to task parameters, while in the second step a fully connected neural network converts the learned task parameters and a clock signal to DMP parameters.

Overall, the DMP framework has the capacity to transform motion trajectories, which is a high-dimension space, to specific motion features, which is a low-dimension latent space, via regression. The motion features are then converted into motor commands by employing a dynamic model as suggested in figure 1. On the other hand, CNN LfD methods utilize convolutions to extract image features from images and then output motor commands via regression. As such, both DMP and CNN frameworks have the capacity to extract low-dimensional features of motion and images, respectively and map them to motor commands. In this paper, we propose an alternative LfD method to compute visuomotor policies by combining the characteristics of both DMPs and CNNs. Figure 3 illustrates our proposed method that utilizes convolutions to extract image features, maps them with regression to DMP motion features, and then produces motor commands with dynamic models. The novelty of the proposed method is that regression is not performed between a high and low dimension space, but between two low-dimension latent spaces, which often yields more successful results [8].

\section{PRELIMINARIES}

In this section, we will summarize the Dynamic Movement Primitives framework as described in [16]. DMPs are a series of differential equations that can describe discrete and rhythmic movements. Since in this paper we are only interested in the behavior of attractor systems, we will only describe the equations for discrete movements. 


$$
\begin{gathered}
\tau \dot{u}=a_{z}\left(\beta_{z}(g-x)-u\right)+\left(g-x_{0}\right) f \\
\tau \dot{x}=u
\end{gathered}
$$

In equations 1 and 2, $x$ and $u$ are the position and velocity, respectively. $\tau$ represents the duration of the trajectory, $x_{0}$ and $g$ are the initial position and target position, respectively. The terms $a_{z}$ and $\beta_{z}$ are control gains selected to make the system critically damped and $f$ acts as the control input that drives the system towards the goal. The forcing term is further defined as:

$$
f(s)=\frac{\sum_{i} w_{i} \psi_{i}(s) s}{\sum_{i} \psi_{i}(s)}
$$

Where $\psi_{i}(s)=\exp \left(-h_{i}\left(s-c_{i}\right)^{2}\right)$ are Gaussian basis functions, with width $h_{i}$ and centers $c_{i}$, while $w_{i}$ are adjustable weights that changes the shape of the Gaussians over the $s$ domain. Note that the forcing term is dependent on $s$, which is derived from the equation:

$$
\tau \dot{s}=\alpha s
$$

The variable $s$ in equation 4 represents a phase variable that starts from time $t=0$ and becomes zero at $t=\tau$. To learn the weights of the forcing term from a demonstration, we record $x(t), u(t)$ and $\dot{u}(t)$ for the duration of the trajectory. From this, we compute $s$ according to equation 4 by setting $\alpha$ so that $s$ becomes zero at the final time. To compute the motor policy, we then estimate $f$ from equation 1 and learn its Gaussian representation by performing regression using the Locally Weighted Regression algorithm (LWR) as suggested in [9]. In short, a DMP behaves like a nonlinear spring damper system [10] that pulls a degree of freedom to a goal position while following a learned trajectory.

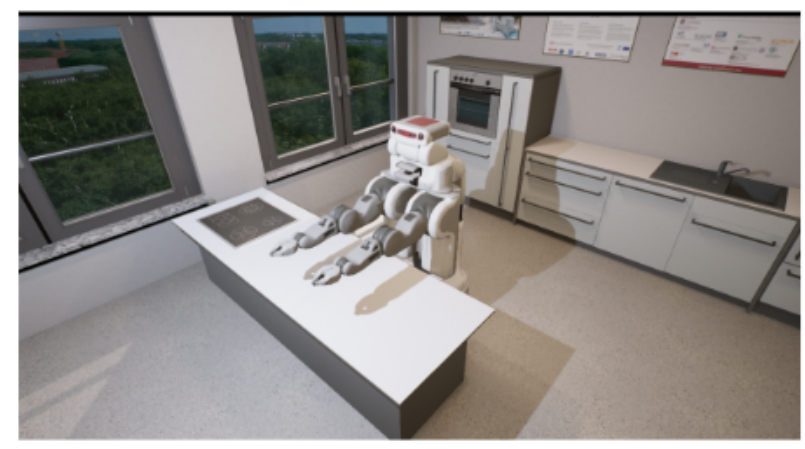

Figure 5: Simulation Environment in Unreal Engine.

\section{SYSTEM ARCHITECTURE}

In this section, we will provide an analysis of the proposed learning method. Figure 3 suggests that the proposed method has two submodules that operate in order. The first one is composed of a CNN that maps raw images and the state of the robot to DMP parameters, while the second sub-module converts the DMP parameters to motor commands. An analytical illustration of the system can be seen in Figure 4.

\subsection{Data Pre-processing}

For training, the learning method requires a dataset $D$ containing multiple demonstrations of each training task. Every demonstration lasts a specific amount of time, which is denoted as the time vector $t=\left[0, . ., t_{i}, . ., t_{f}\right]$, where $t_{f}=\tau$ is the duration of the demonstration and $i$ symbolizes the number of data points per demonstration. Furthermore, we collect observations $o_{i}$, which are RGB images of the demonstration at time $t_{i}$, and we record the robot state vector of joint angle positions $q_{i}$ at time $t_{i}$. The DMP parameters for each task are computed using $q$ and $t$. Specifically, a fixed number of interval recordings $i$ and Gaussians in equation 3 are set. The number of Gaussians, the control gains $a_{z}, b_{z}$, and the variable $\alpha$ are set to constants according to [9], enabling us to derive phase variable $s$ from equation 4 . The starting position $x_{0}$ and goal $g$ is assumed to be the initial $q(t=0)$ and $q(t=T)$ terminal state of the robot for each demonstration. To compute the vector weights $w_{i}$ we used the LWR algorithm [9] as previously expressed. Thus, only the goal $g$, starting pose $x_{0}$, phase $s$, and weights $w_{i}$ are changing in each demonstration. Thus, these variables are the parameters that encode and generalize the joint trajectories of the demonstration.

\subsection{First Sub-module Architecture}

The network's architecture is inspired by [12], [13], and [21] in terms of functionality and composition. We proceed to train the first sub-module after appending the DMP parameters to $D$ and normalizing the dataset. The observations $o_{i}$ are used as input and DMP parameters as output. The RGB images are segmented into 3 channels and their dimension is reduced to $125 \times 125$. Next, they are fed into 4 convolution layers with 16 filters of size 4 and stride of 2 . The output of the last convolution layer is transformed into spatial features with the use of the soft-argmax operator [12], [13]. The spatial image features are then fused with the state of the robot $q_{i}$, which is represented by a vector of joint positions. Note that during the concatenation of the features and the state, we add a bias term to make the image feature dimensions less dominant [12], [13], [4]. The fused dimension is then passed to 3 fully connected layers with 200 units and ReLU activation units. The output of the network represents the DMP parameters, which are used to derive the new forcing term that drives the robot according to equation 1 .

\section{EXPERIMENTAL SECTION}

For the experiments, we have utilized a game engine-based simulation. In this section, we first introduce the simulation and describe the experimental framework. Next, we present test cases to challenge the robustness of the learning method. We continue by elaborating on the data collection method and explain the metrics of evaluation. Finally, we present and discuss the experimental results.

\subsection{Experimental Framework}

Figure 4 provides an illustration of the simulation environment. The environment contains a simulation of a PR2 robot in a virtual kitchen setting. The simulation was developed with Unreal Engine due to its photo-realistic renderings and powerful physics engine. All object in the simulation, including the robot, can be controlled via ROS (using a ROS bridge). The proposed method was implemented with TensorFlow. 

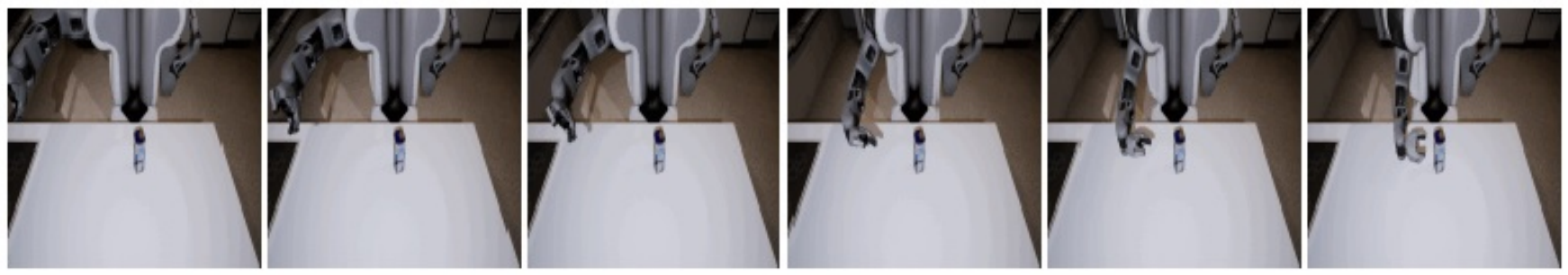

Figure 6: Robot reaches an object from the side.
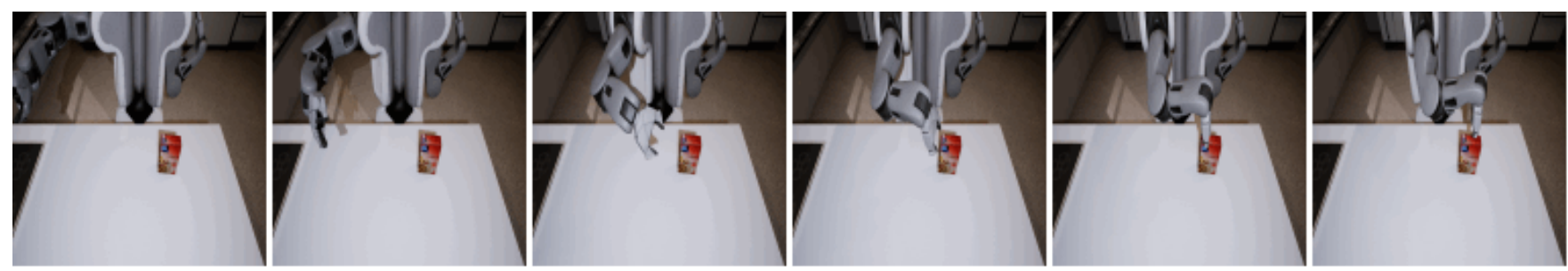

Figure 7: Robot reaches an object from the top.

\subsection{Test Cases}

The training data is acquired with simulated tasks depicting various reaching motions using the robot's right arm. The left arm remains still throughout the experiment. Each demonstration begins with the same starting arm pose while the target objects are placed in novel positions and orientations. The duration of the demonstration, $\tau$, remains constant. This means we can extract the DMP parameters of each demonstration and reduce the number of parameters needed to be learned by the system as the goal $g$ and weight vector $w$ in equation 3 are sufficient for reproducing the movement. The size of the DMP parameter vector (shown in figure 4) is based on the resolution of the DMP forcing term (i.e., the number of Gaussians basis functions) and the degrees of freedom of the manipulator (which is seven in our case with the PR2 robot). The simplification of the representation enables training with mean squared error as opposed to custom cost functions as proposed in [7].

Additional variance is introduced within the dataset by introducing different reaching paths from the initial position of the robot to the object. As Figures 6 and 7 suggest, the robot is able to reach the object from the side or from the top. To be exact, if the observed object is cylindrical in shape, the robot is ordered to approach the object sideways, while if the object is cube-like, the robot approaches to object from the top. The end-to-end network recognizes this automatically and is thus able to infer and select a primitive accordingly.

The trajectory of the demonstration is generated by finding the inverse kinematics solution of a position near the object, and then interpolates the polynomial joint space trajectories leading from the starting joint position. When the joint trajectories are calculated, they are passed to the position controller of the simulated robot and as the robot executes the trajectory, a camera within the simulation records the observations $o_{i}$.

To establish a metric of evaluation, we decided to train an additional CNN. The second neural network has a similar architecture as the one in Figure 4, with the exception that it learns to produce joint velocity commands $d q_{i}$ in every interval based on the observation $o_{i}$ and robot state $q_{i}$. After both networks are trained with data from the same demonstrations, we compare how the robot behaves while being controlled by each network in a set of test cases. In these cases, new objects are introduced and are placed in a set of novel locations with respect to the torso of the robot. For every object and every location, we proceed by comparing how each network commands the robot to reach the object.

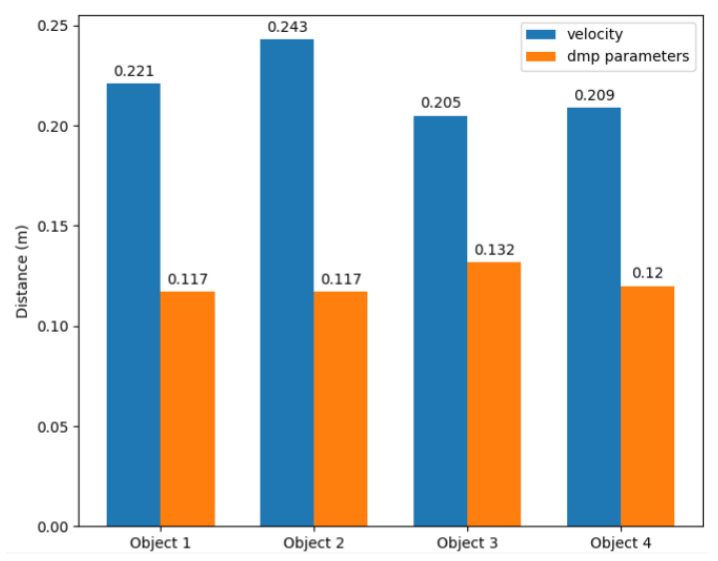

Figure 8: Error between end effector and object. 

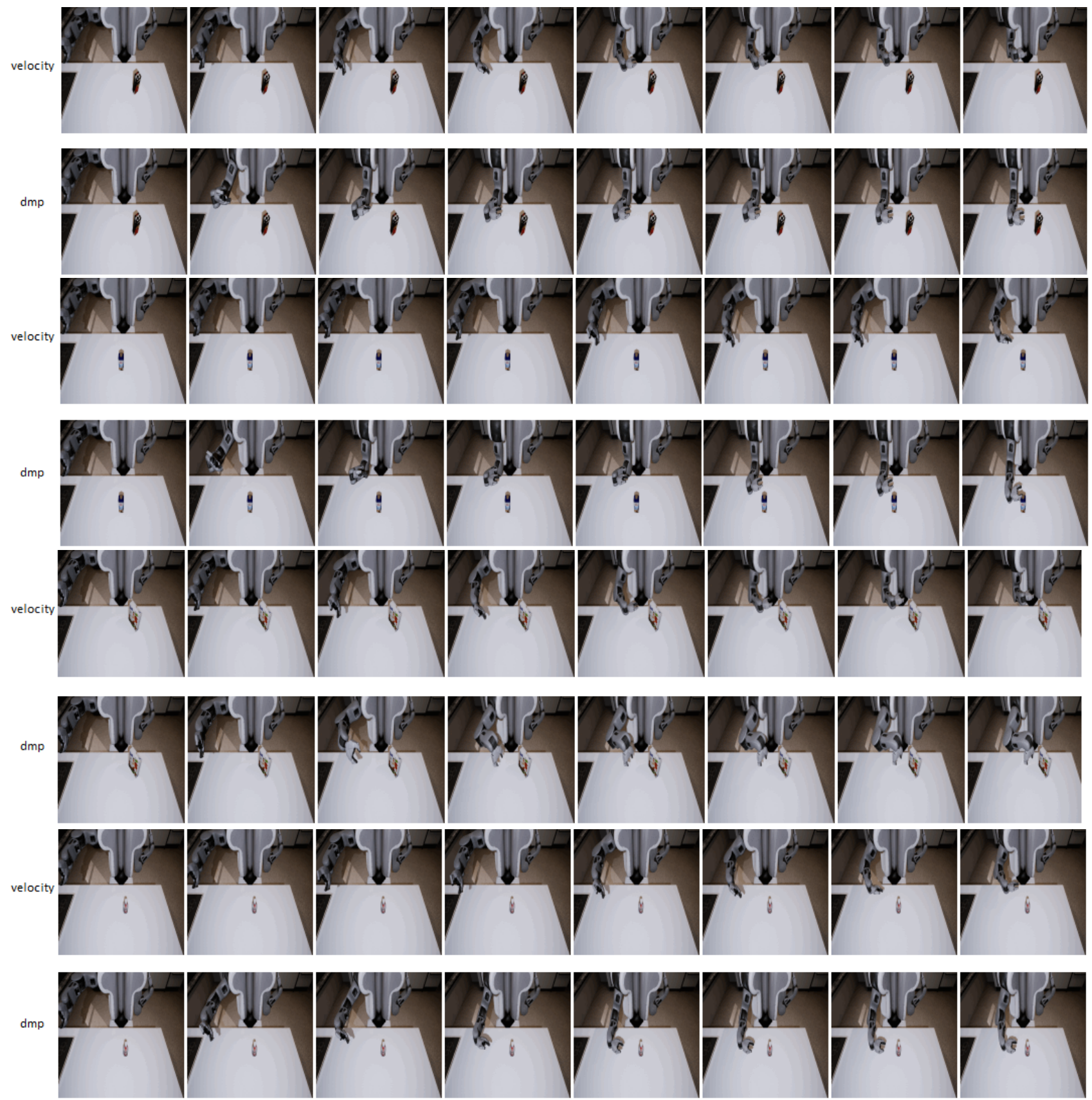

Figure 9: Comparing the behavior of the robot when learning velocities and DMP parameters for 4 different cases. The upper section of every case shows the output when learning velocities, while the lower row depicts the performance of the proposed method.

\subsection{Implementation Details}

As seen from Figures 6 and 7 the robot's right arm always start from $q(t=0)=\left[-90^{\circ},-70^{\circ}, 0^{\circ}, 0^{\circ},-70^{\circ}, 0^{\circ}, 0^{\circ}\right]$. The forcing term of the DMPs is characterized by 20 Gaussian functions. The dataset contains demonstrations of 27 different objects, which are placed in 22 random locations. Each demonstration lasted 4 seconds divided into 100 intervals. During testing, 4 different new objects were placed in the following 10 locations $\delta$ for a total of 40 test cases. As aforementioned, for each test case, we compare how the network that learns DMPs and the network that learns velocities control the robot. The Cartesian positions ( $\mathrm{x}-\mathrm{y}-\mathrm{z})$ and orientations (yaw angle 
in radians) of all the $\delta$ with respect to the torso frame of the robot are as follows:

$$
\begin{gathered}
\delta_{1}=[0.488,0.009,-0.33,-0.0105] \\
\delta_{2}=[0.682,-0.029,-0.33,0.0963] \\
\delta_{3}=[0.608,0.07,-0.33,-0.232] \\
\delta_{4}=[0.615,-0.255,-0.33,-1.522] \\
\delta_{5}=[0.514,0.212,-0.33,-0.6333] \\
\delta_{6}=[0.478,0.147,-0.33,-0.8206] \\
\delta_{7}=[0.627,-0.123,-0.33,0.9057] \\
\delta_{8}=[0.521,-0.285,-0.33,0.3928] \\
\delta_{9}=[0.571,-0.038,-0.33,0.8010] \\
\delta_{10}=[0.627,-0.183,-0.33,0.9661]
\end{gathered}
$$

\subsection{Experimental Results}

The first metric of evaluation is the euclidean distance of the robot's end effector at the terminal state from the center of the object. This metric is selected to establish which system is more successful at guiding the robot near the object. Figure 8 presents a comparison between the two methods with regard to the average error distance in meters from the center of the objects for all 10 locations $\delta$ per object. We notice that the network that learns velocities has an average distance error of $20 \mathrm{~cm}$ in comparison to the proposed method that has an average error of about $11 \mathrm{~cm}$.

Although Figure 8 shows that the proposed method is successful at guiding the robotic arm near the object, we present further comparisons. Figures 9 and 10 provide additional comparisons that show how the robot behaves when controlled by each network. Both Figures illustrate how each network guides the robot when presented with 4 different object for cases $\delta_{4}, \delta_{2}, \delta_{8}$, and $\delta_{1}$ respectively. Figure 9 shows sequences of images that capture the behavior of the robot while it is being controlled by each network individually. Figure 10 presents the equivalent Cartesian trajectories that the robot's end effector followed. A common trend in all cases is that the robot's arm performed an arch shape trajectory when it is controlled by the network that produces velocities. In contrast, the network that learns DMPs guides the robot thought trajectories which are characterized by over-shots and oscillations. Despite this phenomenon, the proposed method retained a sense of goal and direction in comparison to the velocity network. In Figure 10 we highlight that despite the perturbations, the blue trajectory converges to a position near the object's true position, which is denoted as a red dot. Finally, the experiments show that the proposed methodology learned to approach cylindrical objects sideways and cube-like objects from the top. Figure 10 suggests this, because in the third sequence the robot approaches the object from the top, while the other Figures show the robot approaching the object sideways. This pattern occurred in all 40 test cases.
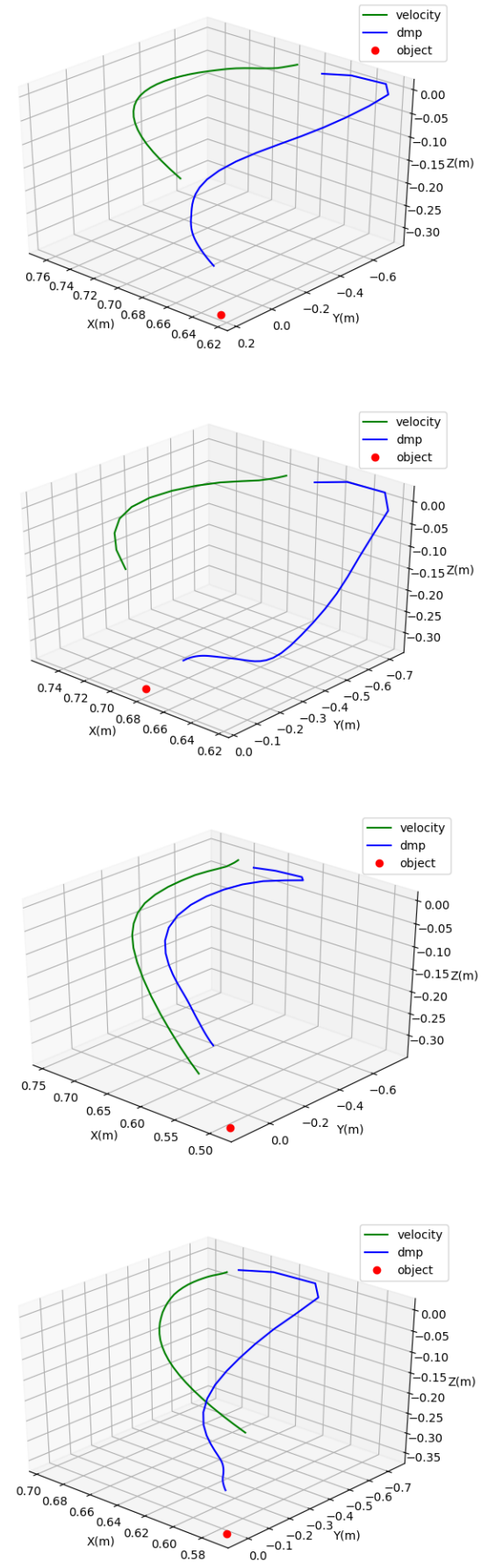

Figure 10: Comparing the Cartesian trajectories of the robot's end effector. The green line represents the output of the velocity network, while the blue one stands for the output of the proposed method. The red dot symbolizes the location of the object. 


\section{CONCLUSION AND FUTURE WORK}

In this paper, we presented a system that learns visuomotor policies by mapping raw images to DMPs. At the core of the system, a Convolutional Neural Network extracts image features and produces motion features. The motion features encode and reproduce motor commands according to the Dynamic Movement Primitives framework. The proposed method was able to guide a robotic arm to a goal location by simply observing raw images. Although the robot was oscillating during the experiments, it learned to perform motor commands that perform the task correctly. However, the testing velocity network interpolated different motor trajectories to produce unsatisfactory motion. This result can be explained by other relevant studies such as [14], [15]. An intuitive explanation is that the geometric interpolation of a top-down and a sideways approach to an object will result in an arching trajectory.

Due to a couple of simplifications in the experimental section, the proposed learning method did not require a custom cost function like [7]. Specifically, each demonstration had the same starting location and duration. As such, the network was able to learn simple motor skills by minimizing the error in the last layer. In the future, we plan to propose a suitable cost function that will allow the network to learn additional DMP parameters. This will enable the network to learn more complicated motor skills and improve its performance by eliminating any oscillations and over-shots, like the ones seen in the experimental results section. Lastly, we will plan to apply quantitative measurements to facilitate the robustness of the proposed method, such as the ones in [18].

\section{ACKNOWLEDGMENTS}

This work was partially funded by Deutsche Forschungsgemeinschaft (DFG) through the Collaborative Research Center 1320, EASE. Joe Cloud has been supported by the NASA Texas Space Grant Graduate Fellowship. Maria Kyrarini and Fillia Makedon have been partially supported by National Science Foundation grants IIS 1565328 and IIP 1719031. This material is based upon work by the authors. Any opinions, findings, conclusions, and recommendations expressed in this paper are those of the author(s) and do not necessarily reflect the views of the National Science Foundation (NSF).

\section{REFERENCES}

[1] Brenna D Argall, Sonia Chernova, Manuela Veloso, and Brett Browning. 2009. A survey of robot learning from demonstration. Robotics and autonomous systems 57, 5 (2009), 469-483.

[2] Aude G Billard, Sylvain Calinon, and Rüdiger Dillmann. 2016. Learning from humans. In Springer handbook of robotics. Springer, 1995-2014.

[3] Sylvain Calinon, Florent Guenter, and Aude Billard. 2007. On learning, representing, and generalizing a task in a humanoid robot. IEEE Transactions on Systems, Man, and Cybernetics, Part B (Cybernetics) 37, 2 (2007), 286-298.

[4] Chelsea Finn, Pieter Abbeel, and Sergey Levine. 2017. Model-agnostic metalearning for fast adaptation of deep networks. In Proceedings of the 34th International Conference on Machine Learning-Volume 70. JMLR. org, 1126-1135.

[5] Chelsea Finn and Sergey Levine. 2017. Deep visual foresight for planning robot motion. In 2017 IEEE International Conference on Robotics and Automation (ICRA). IEEE, 2786-2793

[6] Chelsea Finn, Tianhe Yu, Tianhao Zhang, Pieter Abbeel, and Sergey Levine. 2017. One-shot visual imitation learning via meta-learning. arXiv preprint arXiv:1709.04905 (2017)

[7] Andrej Gams, Aleš Ude, Jun Morimoto, et al. 2018. Deep encoder-decoder networks for mapping raw images to dynamic movement primitives. In 2018 IEEE International Conference on Robotics and Automation (ICRA). IEEE, 1-6.

[8] Geoffrey E Hinton and Ruslan R Salakhutdinov. 2006. Reducing the dimensionality of data with neural networks. science 313, 5786 (2006), 504-507.
[9] Auke Jan Ijspeert, Jun Nakanishi, Heiko Hoffmann, Peter Pastor, and Stefan Schaal. 2013. Dynamical movement primitives: learning attractor models for motor behaviors. Neural computation 25, 2 (2013), 328-373.

[10] Jens Kober, Katharina Mülling, Oliver Krömer, Christoph H Lampert, Bernhard Schölkopf, and Jan Peters. 2010. Movement templates for learning of hitting and batting. In 2010 IEEE International Conference on Robotics and Automation. IEEE, 853-858.

[11] Jens Kober, Andreas Wilhelm, Erhan Oztop, and Jan Peters. 2012. Reinforcement learning to adjust parametrized motor primitives to new situations. Autonomous Robots 33, 4 (2012), 361-379.

[12] Sergey Levine, Chelsea Finn, Trevor Darrell, and Pieter Abbeel. 2016. End-to-end training of deep visuomotor policies. The fournal of Machine Learning Research 17, 1 (2016), 1334-1373.

[13] Sergey Levine, Peter Pastor, Alex Krizhevsky, Julian Ibarz, and Deirdre Quillen. 2018. Learning hand-eye coordination for robotic grasping with deep learning and large-scale data collection. The International fournal of Robotics Research 37 , 4-5 (2018), 421-436.

[14] Katharina Muelling, Jens Kober, and Jan Peters. 2010. Learning table tennis with a mixture of motor primitives. In Humanoid Robots (Humanoids), 2010 10th IEEE-RAS International Conference on. IEEE, 411-416.

[15] Katharina Mülling, Jens Kober, Oliver Kroemer, and Jan Peters. 2013. Learning to select and generalize striking movements in robot table tennis. The International Journal of Robotics Research 32, 3 (2013), 263-279.

[16] Peter Pastor, Heiko Hoffmann, Tamim Asfour, and Stefan Schaal. 2009. Learning and generalization of motor skills by learning from demonstration. In Robotics and Automation, 2009. ICRA'09. IEEE International Conference on. IEEE, 763-768.

[17] Affan Pervez, Yuecheng Mao, and Dongheui Lee. 2017. Learning deep movement primitives using convolutional neural networks. In 2017 IEEE-RAS 17th International Conference on Humanoid Robotics (Humanoids). IEEE, 191-197.

[18] Deirdre Quillen, Eric Jang, Ofir Nachum, Chelsea Finn, Julian Ibarz, and Sergey Levine. 2018. Deep reinforcement learning for vision-based robotic grasping: A simulated comparative evaluation of off-policy methods. In 2018 IEEE International Conference on Robotics and Automation (ICRA). IEEE, 6284-6291.

[19] Stefan Schaal. 1999. Is imitation learning the route to humanoid robots? Trends in cognitive sciences 3, 6 (1999), 233-242.

[20] C Sylvain. 2009. Robot programming by demonstration: A probabilistic approach.

[21] Tianhe Yu, Chelsea Finn, Annie Xie, Sudeep Dasari, Tianhao Zhang, Pieter Abbeel, and Sergey Levine. 2018. One-shot imitation from observing humans via domainadaptive meta-learning. arXiv preprint arXiv:1802.01557 (2018). 